\title{
The Gerber-Shiu Discounted Penalty Function of Sparre Andersen Risk Model with a Constant Dividend Barrier
}

\author{
Yujuan Huang ${ }^{1}$ and Wenguang $\mathbf{Y u}^{2}$ \\ ${ }^{1}$ School of Science, Shandong Jiaotong University, Jinan 250357, China \\ ${ }^{2}$ School of Insurance, Shandong University of Finance and Economics, Jinan 250014, China \\ Correspondence should be addressed to Wenguang Yu; yuwg@mail.sdu.edu.cn
}

Received 19 January 2014; Revised 1 May 2014; Accepted 18 June 2014; Published 15 July 2014

Academic Editor: Guangchen Wang

Copyright (C) 2014 Y. Huang and W. Yu. This is an open access article distributed under the Creative Commons Attribution License, which permits unrestricted use, distribution, and reproduction in any medium, provided the original work is properly cited.

This paper constructs a Sparre Andersen risk model with a constant dividend barrier in which the claim interarrival distribution is a mixture of an exponential distribution and an Erlang(n) distribution. We derive the integro-differential equation satisfied by the Gerber-Shiu discounted penalty function of this risk model. Finally, we provide a numerical example.

\section{The Risk Model}

Consider a Sparre Andersen risk model,

$$
U(t)=u+c t-\sum_{i=1}^{N(t)} X_{i} \text { for } t \geq 0,
$$

where $u \geq 0$ represents the initial capital, $c$ is the insurer's rate of premium income per unit time, and $\{N(t), t \geq 0\}$ is the claim number process representing the number of claims up to time $t$. $\left\{X_{i}, i \geq 1\right\}$ is a sequence of i.i.d. random variables representing the individual claim amounts with distribution function $F(x)$ and density function $f(x)$ with mean $\mu$. We assume that $\{N(t), t \geq 0\}$ and $\left\{X_{i}, i \geq 1\right\}$ are independent. Let $\left\{T_{i}, i \geq 1\right\}$ be sequence i.i.d. random variables, which represent the claim interarrival times, and $T_{i}$ has a density function $K(t)$,

$$
K(t)=\beta_{1} \lambda e^{-\lambda t}+\beta_{2} e^{-\lambda t} \frac{\lambda^{n} t^{n-1}}{(n-1) !}, \quad t \geq 0,
$$

where $n \geq 1$ is a positive integer, $\lambda \geq 0, \beta_{1}, \beta_{2} \geq 0$, and $\beta_{1}+$ $\beta_{2}=1$. We further assume that $c E\left[T_{i}\right]>E\left[X_{i}\right]$ for all $i$, which ensure that $\lim _{t \rightarrow \infty} U(t)=\infty$ almost surely. Throughout the paper we use the convention that $\sum_{i=1}^{0} X_{i}=0$.

In recent years the Sparre Andersen model has been studied extensively. Ruin probabilities and many ruin related quantities such as the marginal and joint defective distributions of the time to ruin, the deficit at ruin, the surplus prior to ruin, and the claim size causing ruin have received considerable attention. Some related results can be found in Cai and Dickson [1], Sun and Yang [2], Gerber and Shiu [3], and Ko [4]. Li and Garrido [5] consider a compound renewal (Sparre Andersen) risk process in the presence of a constant dividend barrier in which the claim waiting times are generalized Erlang(n) distributed. The Sparre Andersen model with phase-type interclaim times has been studied by Ren [6]. Ng and Yang [7] study the ruin probability and the distribution of the severity of ruin in risk models with phasetype claims. Landriault and Willmot [8] study the GerberShiu function in a Sparre Andersen model with general interclaim times. Yang and Zhang [9] study a Sparre Andersen model in which the interclaim times are generalized Erlang(n) distributed. They assume that the premium rate is a step function depending on the current surplus level. Landriault and Sendova [10] generalize the Sparre Andersen dual risk model with Erlang(n) interinnovation times by adding a budget-restriction strategy. Shi and Landriault [11] utilize the multivariate version of Lagrange expansion theorem to obtain a series expansion for the density of the time to ruin under a more general distribution assumption, namely, the combination of $n$ exponentials. Yang and Sendova [12] study the Sparre Andersen dual risk model in which the times 
between positive gains are independently and identically distributed and have a generalized $\operatorname{Erlang}(\mathrm{n})$ distribution.

The barrier strategy was initially proposed by De Finetti [13] for a binomial model. From then on, barrier strategies have been studied in a number of papers and books, including Lin et al. [14], Dickson and Waters [15], Li and Lu [16], Yu [1719], Yao et al. [20], Zhu [21], Tan et al. [22], and references therein for details. The purpose of this paper is to extend some results in Li and Garrido [5] and Yang and Zhang [9]. We study the Sparre Andersen risk model with a constant dividend barrier and the claim interarrival distribution is a mixture of an exponential distribution and an Erlang(n) distribution.

The contents of this paper are organized as follows. Section 2 introduces the risk model. In Section 3, we derive the higher-order integro-differential equation for the GerberShiu discounted penalty function. Finally, in the special case we provide the numerical example in Section 4.

\section{The Risk Model}

Let $U_{b}(t)$ be the surplus process with initial surplus $U_{b}(0)=u$ under the barrier strategy. Thus, it can be expressed as

$$
d U_{b}(t)= \begin{cases}c d t-d S(t) & U_{b}(t)<b \\ -d S(t) & U_{b}(t) \geq b\end{cases}
$$

where $S(t)=\sum_{i=1}^{N(t)} X_{i}$. Define $T_{b}=\inf \left\{t: U_{b}(t)<0\right\}$ to be the first time that the surplus becomes negative. The stopping time $T_{b}$ is referred to as the time of ruin. Let $\psi_{b}(u)=\operatorname{Pr}\left(T_{b}<\right.$ $\infty)$ be the ruin probability.

In this paper, we will study the time of ruin $T_{b}$ and its related functions such as the surplus before ruin $U_{b}\left(T_{b}-\right)$ and the deficit at ruin $\left|U_{b}\left(T_{b}\right)\right|$. By using a renewal equation approach, we will be able to get a number of analytic and probabilistic properties of these quantities. Our analysis will involve the Gerber-Shiu discounted penalty function that is defined below.

Let $\omega(x, y), 0 \leq x, y<\infty$, be a nonnegative function. For $\delta \geq 0$, define

$$
\begin{array}{r}
m_{b}(u)=E\left[e^{-\delta T_{b}} \omega\left(U\left(T_{b}-\right),\left|U\left(T_{b}\right)\right|\right)\right. \\
\left.I\left(T_{b}<\infty\right) \mid U(0)=u\right],
\end{array}
$$

where $I(\cdot)$ is the indicator function, $I\left(T_{b}<\infty\right)=1$ if $T_{b}<$ $\infty$, and $I\left(T_{b}<\infty\right)=0$ otherwise. The function $m_{b}(u)$ in (4) is useful for deriving results in connection with joint and marginal distributions of $T_{b}, U_{b}\left(T_{b}-\right)$ and $\left|U_{b}\left(T_{b}\right)\right|$. While $\delta$ may be interpreted as a force of interest, function (4) may also be viewed in terms of a Laplace transform with $\delta$ serving as the argument. In particular, if we let $\omega(x, y)=1,(4)$ is the Laplace transform of the time of ruin $T_{b}$. If we let $\delta=0$ and $\omega(x, y)=1$, then $m_{b}(u)$ becomes the ruin probability $\psi(u)$. If we let $\delta=0$ and $\omega(x, y)=I\left(x \leq z_{1}\right) I\left(y \leq z_{2}\right)$, (4) becomes the joint $\mathrm{df}$ of the surplus before ruin and the deficit at ruin. Furthermore, if $\delta=0$ and $\omega(x, y)=x_{1}^{n}$, we obtain the $n$th moment of the surplus before ruin. Likewise, if $\delta=0$ and $\omega(x, y)=x_{2}^{n}$, we obtain the $n$th moment of the deficit at ruin.
For other functions of interest, see Gerber and Shiu [23] and Lin and Willmot [24]. Let $f^{*}$ denote the Laplace transform of the function $f$, that is, $f^{*}(s)=\int_{0}^{\infty} e^{-s x} f(x) d x$.

\section{An Integro-Differential Equation}

In this section, we show $m_{b}(u)$ satisfies a higher-order integro-differential equation.

Lemma 1. Assume $s>u$; then $H(u, s)=K((s-$ $u) / c) e^{-\delta((s-u) / c)}$ satisfies the following differential equation:

$$
\begin{gathered}
\sum_{j=0}^{n-1} C_{n-1}^{j} c^{j}(-\lambda-\delta)^{n-1-j} \frac{\partial^{j} H(u, s)}{\partial u^{j}} \\
=(-1)^{n-1} \beta_{2} \lambda^{n} e^{-(\lambda+\delta)((s-u) / c)},
\end{gathered}
$$

with the boundary conditions when $s=u$,

$$
\begin{gathered}
\frac{\partial^{k} H(u, s)}{\partial u^{k}}=\beta_{1} \lambda\left(\frac{\lambda+\delta}{c}\right)^{k}, \quad k=0,1,2, \ldots, n-2, \\
\frac{\partial^{n-1} H(u, s)}{\partial u^{n-1}}=\beta_{1} \lambda\left(\frac{\lambda+\delta}{c}\right)^{n-1}+\beta_{2} \lambda^{n}\left(-\frac{1}{c}\right)^{n-1} .
\end{gathered}
$$

Proof. Note that $H(u, s)=\left[\beta_{1} \lambda+\left(\lambda^{n} \beta_{2} /(n-\right.\right.$ $\left.1) !)((\lambda+\delta) / c)^{n-1}\right] e^{-(\lambda+\delta)((s-u) / c)}$. Taking derivative with respect to variable $u$ for $k$ times and by induction, we can obtain

$$
\begin{aligned}
& \frac{\partial^{k} H(u, s)}{\partial u^{k}} \\
& =-\sum_{j=0}^{k-1} C_{k}^{j}\left(-\frac{\lambda+\delta}{c}\right)^{k-j} \frac{\partial^{j} H(u, s)}{\partial u^{j}} \\
& +\frac{\beta_{2} \lambda^{n}}{(n-1-k) !}\left(\frac{s-u}{c}\right)^{n-1-k}\left(-\frac{1}{c}\right)^{k} e^{-(\lambda+\delta)((s-u) / c)}, \\
& \quad 0 \leq k \leq n-1 .
\end{aligned}
$$

When $k=n-1$, one gets (5). On substituting $s=u$ in (7), we get the boundary conditions.

Theorem 2. The Gerber-Shiu discounted penalty function $m_{b}(u)$ satisfies the higher-order integro-differential equation

$$
\begin{aligned}
\sum_{k=0}^{n} C_{n}^{k} c^{k}(-\lambda-\delta)^{n-k} \frac{d^{k} m_{b}(u)}{d u^{k}} & \\
= & {\left[\beta_{2}(-\lambda)^{n}+\beta_{1}(-\lambda)(-\lambda-\delta)^{n-1}\right] } \\
& \times \int_{0}^{\infty} m_{b}(u-x) d F(x) \\
& -\beta_{1} \lambda \sum_{k=1}^{n-1} C_{n-1}^{k} c^{k}(-\lambda-\delta)^{n-k-1} \frac{d^{k}}{d u^{k}} \\
& \times\left(\int_{0}^{\infty} m_{b}(u-x) d F(x)\right) .
\end{aligned}
$$


Mathematical Problems in Engineering

3

Proof. Let $t$ be the time of the first claim and let $x$ be the amount of the claim. There are two possibilities. First, $t<$ $(b-u) / c$ and the surplus has not yet reached the barrier. In this case, the surplus immediately before time $t$ is $u+c t$. Second, $t \geq(b-u) / c$ and the surplus immediately before time $t$ is $b$. And since the "probability" that the claim occurs at time $t$ is $K(t) d t$ and the "probability" of the claim amount being $x$ is $d F(x)$, we have, for $0 \leq u \leq b$,

$$
\begin{aligned}
m_{b}(u)= & \int_{0}^{(b-u) / c} K(t) e^{-\delta t} \\
& \times\left[\int_{0}^{u+c t} m_{b}(u+c t-x) d F(x)\right. \\
& \left.+\int_{u+c t}^{\infty} w(u+c t, x-u-c t) d F(x)\right] d t \\
+ & \int_{((b-u) / c)}^{\infty} K(t) e^{-\delta t} \\
& \times\left[\int_{0}^{b} m_{b}(b-x) d F(x)\right. \\
& \left.+\int_{b}^{\infty} w(b, x-b) d F(x)\right] d t .
\end{aligned}
$$

Using the substitution $s=u+c t$, we have

$$
\begin{aligned}
m_{b}(u)= & \int_{u}^{b} K\left(\frac{s-u}{c}\right) e^{-\delta((s-u) / c)} \\
& \times\left[\int_{0}^{s} m_{b}(s-x) d F(x)\right. \\
& \left.+\int_{s}^{\infty} w(s, x-s) d F(x)\right] \frac{1}{c} d s \\
+ & \int_{b}^{\infty} K\left(\frac{s-u}{c}\right) e^{-\delta((s-u) / c)} \\
& \times\left[\int_{0}^{b} m_{b}(b-x) d F(x)\right. \\
& \left.+\int_{b}^{\infty} w(b, x-b) d F(x)\right] \frac{1}{c} d s
\end{aligned}
$$

which implies that

$$
\begin{aligned}
c m_{b}(u)= & \int_{u}^{b} H(u, s) \int_{0}^{\infty} m_{b}(s-x) d F(x) d s \\
& +\int_{b}^{\infty} H(u, s) \int_{0}^{\infty} m_{b}(b-x) d F(x) d s,
\end{aligned}
$$

where $H(u, s)$ is defined in Lemma 1. Differentiating the above equation $k$ times and using condition (6) yield

$$
\begin{aligned}
& c \frac{d^{k} m_{b}(u)}{d u^{k}} \\
& =-\beta_{1} \lambda \sum_{i=0}^{k-1}\left(\frac{\lambda+\delta}{c}\right)^{k-1-i} \frac{d^{i}}{d u^{i}} \int_{0}^{\infty} m_{b}(u-x) d F(x) \\
& \quad+\int_{u}^{b} \frac{\partial^{k} H(u, s)}{\partial u^{k}} \int_{0}^{\infty} m_{b}(s-x) d F(x) d s \\
& \quad+\int_{b}^{\infty} \frac{\partial^{k} H(u, s)}{\partial u^{k}} \int_{0}^{\infty} m_{b}(b-x) d F(x) d s .
\end{aligned}
$$

Multiplying (12) by $c^{k}(-\lambda-\delta)^{n-1-k} C_{n-1}^{k}$ for $k=0,1,2, \ldots, n-$ 1 , then adding up these equations, and using (5), we obtain

$$
\begin{aligned}
\sum_{k=0}^{n-1} C_{n-1}^{k}(-\lambda-\delta)^{n-1-k} c^{k+1} \frac{d^{k} m_{b}(u)}{d u^{k}} \\
=\beta_{2} \lambda^{n}(-1)^{n-1} \int_{u}^{b} e^{-(\lambda+\delta)((s-u) / c)} \\
\quad \times \int_{0}^{\infty} m_{b}(s-x) d F(x) d s \\
+\beta_{2} \lambda^{n}(-1)^{n-1} \int_{b}^{\infty} e^{-(\lambda+\delta)((s-u) / c)} \\
\quad \times \int_{0}^{\infty} m_{b}(b-x) d F(x) d s \\
\quad-\beta_{1} \lambda \sum_{k=1}^{n-1} C_{n-1}^{k}(-\lambda-\delta)^{n-1-k} c^{k} \\
\quad \times\left[\sum_{i=0}^{k-1}\left(\frac{\lambda+\delta}{c}\right)^{k-1-i} \frac{d^{i}}{d u^{i}}\left(\int_{0}^{\infty} m_{b}(u-x) d F(x)\right)\right]
\end{aligned}
$$

Differentiating (13) again, we have

$$
\begin{gathered}
\sum_{k=0}^{n-1} C_{n-1}^{k}(-\lambda-\delta)^{n-1-k} c^{k+1} \frac{d^{k+1} m_{b}(u)}{d u^{k+1}} \\
=(-1)^{n} \beta_{2} \lambda^{n} \int_{0}^{\infty} m_{b}(u-x) d F(x) \\
-\beta_{1} \lambda \sum_{k=1}^{n-1} C_{n-1}^{k}(-\lambda-\delta)^{n-1-k} c^{k}
\end{gathered}
$$




$$
\begin{aligned}
& \times\left[\sum_{i=0}^{k-1}\left(\frac{\lambda+\delta}{c}\right)^{k-1-i} \frac{d^{i+1}}{d u^{i+1}}\left(\int_{0}^{\infty} m_{b}(u-x) d F(x)\right)\right] \\
& +\frac{\lambda+\delta}{c} \beta_{2} \lambda^{n}(-1)^{n-1} \\
& \times\left[\int_{u}^{b} e^{-(\lambda+\delta)((s-u) / c)} \int_{0}^{\infty} m_{b}(s-x) d F(x) d s\right. \\
& \left.+\int_{b}^{\infty} e^{-(\lambda+\delta)((s-u) / c)} \int_{0}^{\infty} m_{b}(b-x) d F(x) d s\right]
\end{aligned}
$$

which, together with (13), implies

$$
\begin{aligned}
\sum_{k=0}^{n-1} C_{n-1}^{k} c^{k+1}(-\lambda-\delta)^{n-1-k} \frac{d^{k+1} m_{b}(u)}{d u^{k+1}} & +\sum_{k=0}^{n-1} C_{n-1}^{k} c^{k}(-\lambda-\delta)^{n-k} \frac{d^{k} m_{b}(u)}{d u^{k}} \\
= & -\beta_{1} \lambda \sum_{k=1}^{n-1} C_{n-1}^{k}(-\lambda-\delta)^{n-1-k} c^{k} \\
& \times\left[\sum_{i=0}^{k-1}\left(\frac{\lambda+\delta}{c}\right)^{k-1-i} \frac{d^{i+1}}{d u^{i+1}}\left(\int_{0}^{\infty} m_{b}(u-x) d F(x)\right)\right] \\
& -\beta_{1} \lambda \sum_{k=1}^{n-1} C_{n-1}^{k}(-\lambda-\delta)^{n-k} c^{k-1} \\
& \times\left[\sum_{i=0}^{k-1}\left(\frac{\lambda+\delta}{c}\right)^{k-1-i} \frac{d^{i}}{d u^{i}}\left(\int_{0}^{\infty} m_{b}(u-x) d F(x)\right)\right] \\
& +(-1)^{n} \beta_{2} \lambda^{n} \int_{0}^{\infty} m_{b}(u-x) d F(x)
\end{aligned}
$$

Moreover, note that

$$
\begin{aligned}
& \sum_{k=0}^{n-1} C_{n-1}^{k} c^{k+1}(-\lambda-\delta)^{n-1-k} \frac{d^{k+1} m_{b}(u)}{d u^{k+1}} \\
& =c^{n} \frac{d^{n} m_{b}(u)}{d u^{n}}+\sum_{k=1}^{n-1} C_{n-1}^{k-1} c^{k}(-\lambda-\delta)^{n-k} \frac{d^{k} m_{b}(u)}{d u^{k}} \\
& \sum_{k=0}^{n-1} C_{n-1}^{k} c^{k}(-\lambda-\delta)^{n-k} \frac{d^{k} m_{b}(u)}{d u^{k}} \\
& =(-\lambda-\delta)^{n} m_{b}(u)+\sum_{k=1}^{n-1} C_{n-1}^{k} c^{k}(-\lambda-\delta)^{n-k} \frac{d^{k} m_{b}(u)}{d u^{k}}
\end{aligned}
$$

So, it follows from (16) that

$$
\begin{gathered}
\sum_{k=0}^{n-1} C_{n-1}^{k} c^{k+1}(-\lambda-\delta)^{n-1-k} \frac{d^{k+1} m_{b}(u)}{d u^{k+1}} \\
+\sum_{k=0}^{n-1} C_{n-1}^{k} c^{k}(-\lambda-\delta)^{n-k} \frac{d^{k} m_{b}(u)}{d u^{k}} \\
=\sum_{k=0}^{n} C_{n}^{k} c^{k}(-\lambda-\delta)^{n-k} \frac{d^{k} m_{b}(u)}{d u^{k}}
\end{gathered}
$$

and thus the result follows from (15) and (17).

Remark 3. Letting $\beta_{1}=0, \beta_{2}=1, n=2$ in (8), we get the integro-differential equation for Erlang (2) risk model with a constant dividend barrier of Li and Garrido [5].

Remark 4. Letting $\beta_{1}=0, \beta_{2}=1, n=2, b=\infty$ in (8), we obtain the integro-differential equation for Erlang (2) risk model with no dividend barrier, which has been considered in Dickson and Hipp [25].

Remark 5. Letting $n=1, b=\infty$ in (8), we derive the integrodifferential equation for classical risk model. For details, see Gerber and Shiu [23].

Remark 6. Letting $n=1$, the case has been studied in Lin et al. [14].

Remark 7. Letting $b=\infty$, the case has been studied in Zhao and Yin [26].

Theorem 8. The Laplace transform of $m_{b}(u)$ is

$$
\begin{aligned}
& m_{b}^{*}(s) \\
& \quad=\frac{A \int_{0}^{\infty} e^{-s u} \int_{u}^{\infty} \omega(u, x-u) d F(x) d u+G(s)+D(s)}{(s c-\lambda-\delta)^{n}-\left[\beta_{2}(-\lambda)^{n}-\lambda \beta_{1}(s c-\lambda-\delta)^{n-1}\right] f^{*}(s)},
\end{aligned}
$$

where

$$
\begin{aligned}
A & =\beta_{2}(-\lambda)^{n}+\beta_{1}(-\lambda)(-\lambda-\delta)^{n-1}, \\
G(s) & =\sum_{k=1}^{n-1} \sum_{j=0}^{k-1} C_{n}^{k}(-\lambda-\delta)^{n-k} c^{k} s^{k-1-j} m_{b}^{(j)}(0), \\
D(s)= & \beta_{1} \lambda \sum_{k=2}^{n-1} C_{n-1}^{k}(-\lambda-\delta)^{n-1-k} c^{k} \\
& \times \sum_{j=1}^{k-1} s^{k-1-j} \sum_{l=0}^{j-1} m_{b}^{(l)}(0) f^{(j-1-l)}(0) \\
& -\beta_{1} \lambda \sum_{k=1}^{n-1} C_{n-1}^{k}(-\lambda-\delta)^{n-1-k} c^{k} \\
& \times \int_{0}^{\infty} e^{-s u}\left[\frac{d^{k}}{d u^{k}} \int_{u}^{\infty} \omega(u, x-u) d F(x)\right] d u .
\end{aligned}
$$


Proof. It is easy to see that

$$
\begin{aligned}
& \int_{0}^{\infty} e^{-s u} \frac{d^{k} m_{b}(u)}{d u^{k}} d u=s^{k} m_{b}^{*}(s)-\sum_{j=0}^{k-1} s^{k-1-j} m_{b}^{(j)}(0), \\
& \int_{0}^{\infty} e^{-s u} \int_{0}^{\infty} m_{b}(u-x) d F(x) d u \\
& =\int_{0}^{\infty} e^{-s u} \int_{0}^{u} m_{b}(u-x) d F(x) d u \\
& \quad+\int_{0}^{\infty} e^{-s u} \int_{u}^{\infty} \omega(u, x-u) d F(x) d u \\
& =s m_{b}^{*}(s) f^{*}(s)+\int_{0}^{\infty} e^{-s u} \int_{u}^{\infty} \omega(u, x-u) d F(x) d u,
\end{aligned}
$$

$$
\begin{aligned}
& \int_{0}^{\infty} e^{-s u} \frac{d^{k}}{d u^{k}}\left(\int_{0}^{\infty} m_{b}(u-x) d F(x)\right) d u \\
& =\int_{0}^{\infty} e^{-s u} \frac{d^{k}}{d u^{k}}\left(\int_{0}^{u} m_{b}(u-x) d F(x)\right) d u \\
& \quad+\int_{0}^{\infty} e^{-s u} \frac{d^{k}}{d u^{k}}\left(\int_{u}^{\infty} \omega(u, x-u) d F(x)\right) d u \\
& =s^{k} m_{b}^{*}(s) f^{*}(s)-\sum_{j=1}^{k-1} s^{k-1-j} \sum_{l=0}^{j-1} m_{b}^{(l)}(0) f^{(j-1-l)}(0) \\
& \quad+\int_{0}^{\infty} e^{-s u} \frac{d^{k}}{d u^{k}}\left(\int_{u}^{\infty} \omega(u, x-u) d F(x)\right) d u .
\end{aligned}
$$

Taking the Laplace transform on both sides of (8), and together with (20), (21), and (22), we have

$$
\begin{aligned}
\sum_{k=0}^{n} C_{n}^{k} c^{k}(-\lambda-\delta)^{n-k}\left(s^{k} m_{b}^{*}(s)-\sum_{j=0}^{k-1} s^{k-1-j} m_{b}^{(j)}(0)\right) \\
=\left[\beta_{2}(-\lambda)^{n}+\beta_{1}(-\lambda)(-\lambda-\delta)^{n-1}\right] \\
\quad \times\left[m_{b}^{*}(s) f^{*}(s)+\int_{0}^{\infty} e^{-s u} \int_{u}^{\infty} \omega(u, x-u) d F(x) d u\right] \\
-\beta_{1} \lambda\left[(s c-\lambda-\delta)^{n-1}-(-\lambda-\delta)^{n-1}\right] m_{b}^{*}(s) f^{*}(s) \\
+\beta_{1} \lambda \sum_{k=2}^{n-1} C_{n-1}^{k} c^{k}(-\lambda-\delta)^{n-1-k} \\
\quad \times \sum_{j=1}^{k-1} s^{k-1-j} \sum_{l=0}^{j-1} m_{b}^{(l)}(0) f^{(j-1-l)}(0) \\
\quad-\beta_{1} \lambda \sum_{k=1}^{n-1} C_{n-1}^{k} c^{k}(-\lambda-\delta)^{n-1-k} \\
\times \int_{0}^{\infty} e^{-s u}\left[\frac{d^{k}}{d u^{k}} \int_{u}^{\infty} \omega(u, x-u) d F(x)\right] d u
\end{aligned}
$$

which implies (8).
Lemma 9. Let $\delta$ be strictly positive and $n$ is a positive integer; then the equation

$$
\begin{aligned}
(s c-\lambda-\delta)^{n}= & f^{*}(s)\left[\beta_{2}(-\lambda)^{n}+\beta_{1}(-\lambda)(-\lambda-\delta)^{n-1}\right] \\
& -f^{*}(s) \lambda \beta_{1}\left[(s c-\lambda-\delta)^{n-1}-(-\lambda-\delta)^{n-1}\right]
\end{aligned}
$$

has exact $n$ roots $s_{l}(\delta)$ with $\operatorname{Re}\left(s_{l}(\delta)\right)>0(l=1,2,3, \ldots, n)$.

Proof. When $s=0$, we have

$$
\left|\left[\beta_{2}(-\lambda)^{n}+\beta_{1}(-\lambda)(-\lambda-\delta)^{n-1}\right] f^{*}(0)\right|<\left|(-\lambda-\delta)^{n}\right| .
$$

So for $\rho>0$ sufficiently big, the inequality

$$
\begin{aligned}
& \quad\left|(s c-\lambda-\delta)^{n}\right| \\
& >\mid\left[\beta_{2}(-\lambda)^{n}+\beta_{1}(-\lambda)(-\lambda-\delta)^{n-1}\right. \\
& \left.\quad-\lambda \beta_{1}(s c-\lambda-\delta)^{n-1}+\lambda \beta_{1}(-\lambda-\delta)^{n-1}\right] f^{*}(s) \mid
\end{aligned}
$$

holds on the imaginary axis and on the semicircle $\{s \in$ $£, \operatorname{Re}(s)>0,|s|=\rho\}$. By Rouches theorem (20) has exact $n$ roots on the right-half plane.

\section{Numerical Illustration for Ruin Probability}

In this section, we give the numerical illustration for $m_{b}(u)$ when the claim number process has Erlang $(2)$ process $\left(\beta_{1}=\right.$ $\left.0, \beta_{2}=1, n=2\right), \delta=0$ and $w(x, y)=1$. At this time, $m_{b}(u)$ turns to ruin probability $\psi_{b}(u)$. By conditioning on the time of the first claim we have, for $0 \leq u \leq b$,

$$
\begin{aligned}
m_{b}(u)= & \int_{0}^{((b-u) / c)} K_{1}(t) \gamma_{b}(u+c t) d t \\
& +\int_{((b-u) / c)}^{\infty} K_{1}(t) \gamma_{b}(b) d t
\end{aligned}
$$

where

$$
\gamma_{b}(t)=\int_{0}^{t} m_{b}(t-x) d F(x)+1-F(t) .
$$

Substituting $K_{1}(t)=\lambda^{2} t e^{-\lambda t}$ into (27), we obtain

$$
\begin{aligned}
m_{b}(u)= & \left(\frac{\lambda}{c}\right)^{2} \int_{u}^{b}(t-u) e^{-(\lambda / c)(t-u)} \gamma_{b}(t) d t \\
& +\gamma_{b}(b) e^{-(\lambda / c)(t-u)}\left[1+\frac{\lambda}{c}(b-u)\right] .
\end{aligned}
$$

Differentiating (29) with respect to $u$, we have, for $0 \leq u \leq b$,

$$
\begin{aligned}
m_{b}^{\prime}(u)= & \frac{\lambda}{c} m_{b}(u)-\left(\frac{\lambda}{c}\right)^{2} \int_{u}^{b} e^{-(\lambda / c)(t-u)} \gamma_{b}(t) d t \\
& -\frac{\lambda \gamma_{b}(b)}{c} e^{-(\lambda / c)(b-u)} .
\end{aligned}
$$


Differentiating (30) again with respect to $u$, we have

$$
\begin{aligned}
m_{b}^{\prime \prime}(u)= & \frac{\lambda}{c} m_{b}^{\prime}(u) \\
& -\frac{\lambda}{c}\left[\left(\frac{\lambda}{c}\right)^{2} \int_{u}^{b} e^{-(\lambda / c)(t-u)} \gamma_{b}(t) d t\right. \\
& \left.+\frac{\lambda}{c} \gamma_{b}(b) e^{-(\lambda / c)(b-u)}\right] \\
& +\left(\frac{\lambda}{c}\right)^{2} \gamma_{b}(u) .
\end{aligned}
$$

Suppose the claim size distribution is exponential. Let $F(x)=$ $1-e^{-\alpha x}, \alpha>0$; then substituting (30) into (31), we have

$$
\begin{aligned}
m_{b}^{\prime \prime}(u)= & \frac{2 \lambda}{c} m_{b}^{\prime}(u)-\left(\frac{\lambda}{c}\right)^{2} m_{b}(u) \\
& +\left(\frac{\lambda}{c}\right)^{2} \alpha e^{-\alpha u} \int_{0}^{u} m_{b}(t) e^{-\alpha t} d t+\left(\frac{\lambda}{c}\right)^{2} e^{-\alpha u}
\end{aligned}
$$

Differentiating (32) with respect to $u$, we have

$$
\begin{aligned}
m_{b}^{\prime \prime \prime}(u)= & \frac{2 \lambda}{c} m_{b}^{\prime \prime}(u)-\left(\frac{\lambda}{c}\right)^{2} m_{b}^{\prime}(u) \\
& -\left(\frac{\lambda}{c}\right)^{2} \alpha^{2} e^{-\alpha u} \int_{0}^{u} m_{b}(t) e^{-\alpha t} d t \\
& +\left(\frac{\lambda}{c}\right)^{2} \alpha m_{b}(u)-\left(\frac{\lambda}{c}\right)^{2} \alpha e^{-\alpha u} .
\end{aligned}
$$

$(32) \times \alpha+(33)$ implies

$$
m_{b}^{\prime \prime \prime}(u)+\left(\alpha-\frac{2 \lambda}{c}\right) m_{b}^{\prime \prime}(u)+\frac{\lambda^{2}-2 \alpha c \lambda}{c^{2}} m_{b}^{\prime}(u)=0 .
$$

This is a three-order differential equation with constant coefficients, so we can carry on the numerical solution. Suppose $\alpha=10000, c=200, \lambda=0.0001, b=20$; then by the Matlab, we obtain the curve of ruin probability (see Figure 1). As is known to all ruin must occur under the constant dividend barrier. From Figure 1, we know that ruin probability $\psi_{b}(u)$ is an increasing function of the initial surplus $u$ (convex function) and the function value of 1 is its asymptote.

\section{Conflict of Interests}

The authors declare that there is no conflict of interests regarding the publication of this paper.

\section{Acknowledgments}

The authors would like to thank an anonymous referee for the valuable comments and suggestions that improved the paper presentation. This work was supported by National Natural

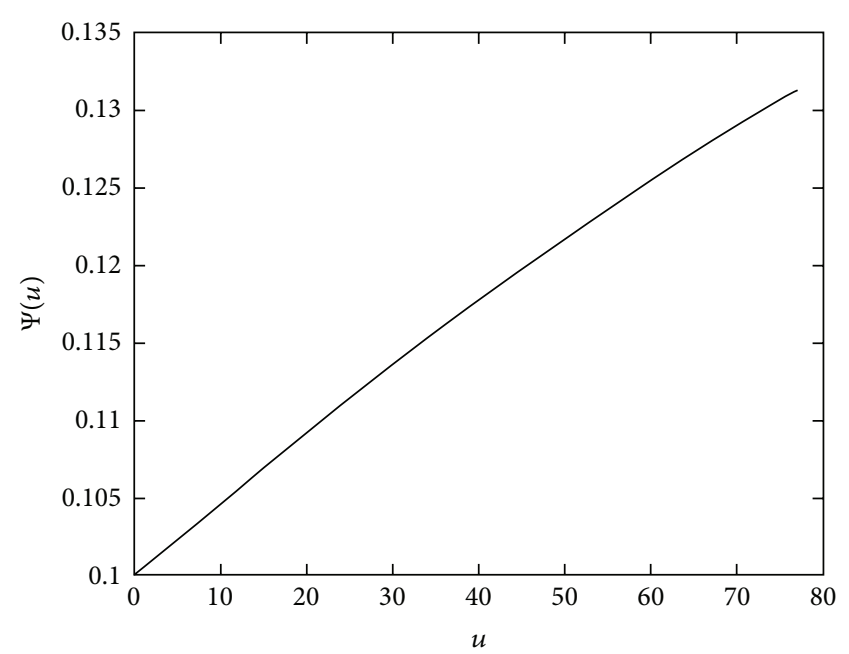

FIGURE 1: The curve of ruin probability.

Science Foundation of China (no. 11301303), Natural Science Foundation of Shandong Province (nos. ZR2012AQ013 and ZR2010GL013), Humanities and Social Sciences Project of the Ministry of Education of China (no. 14YJA630088, no. 13YJC630150, no. 10YJC630092, and no. 09YJC910004), and the Doctorate Scientific Research Foundation of Shandong Jiaotong University.

\section{References}

[1] J. Cai and D. C. M. Dickson, "Upper bounds for ultimate ruin probabilities in the Sparre Andersen model with interest," Insurance: Mathematics \& Economics, vol. 32, no. 1, pp. 61-71, 2003.

[2] L. J. Sun and H. L. Yang, "On the joint distributions of surplus immediately before ruin and the deficit at ruin for Erlang(2) risk processes," Insurance: Mathematics \& Economics, vol. 34, no. 1, pp. 121-125, 2004.

[3] H. U. Gerber and E. S. W. Shiu, "The time value of ruin in a Sparre Andersen model," North American Actuarial Journal, vol. 9, no. 2, pp. 49-69, 2005.

[4] B. Ko, "Discussion of the discounted joint distribution of the surplus prior to ruin and the deficit at ruin in a Sparre Andersen model," North American Actuarial Journal, vol. 11, no. 3, pp. 136137, 2007.

[5] S. Li and J. Garrido, "On a class of renewal risk models with a constant dividend barrier," Insurance: Mathematics \& Economics, vol. 35, no. 3, pp. 691-701, 2004.

[6] J. Ren, "The discounted joint distribution of the surplus prior to ruin and the deficit at ruin in a Sparre Andersen model," North American Actuarial Journal, vol. 11, no. 3, pp. 128-136, 2007.

[7] A. C. Y. Ng and H. Yang, "Lundberg-type bounds for the joint distribution of surplus immediately before and at ruin under the Sparre Andersen model," North American Actuarial Journal, vol. 9, no. 2, pp. 85-107, 2005.

[8] D. Landriault and G. E. Willmot, "On the Gerber- Shiu discounted penalty function in the Sparre Andersen model with an arbitrary inter-claim time distribution," Insurance: Mathematics \& Economics, vol. 42, no. 2, pp. 600-608, 2008. 
[9] H. Yang and Z. Zhang, "Gerber-Shiu discounted penalty function in a Sparre Andersen model with multi-layer dividend strategy," Insurance: Mathematics \& Economics, vol. 42, no. 3, pp. 984-991, 2008.

[10] D. Landriault and K. P. Sendova, "A direct approach to a firstpassage problem with applications in risk theory," Stochastic Models, vol. 27, no. 3, pp. 388-406, 2011.

[11] T. X. Shi and D. Landriault, "Distribution of the time to ruin in some Sparre Andersen risk models," ASTIN Bulletin, vol. 43, no. 1, pp. 39-59, 2013.

[12] C. Yang and K. P. Sendova, "The ruin time under the SparreAndersen dual model," Insurance: Mathematics \& Economics, vol. 54, no. 1, pp. 28-40, 2014.

[13] B. De Finetti, "Su un'impostazione alternativa dell teoria colletiva del rischio," in Proceedings of the Transactions of the 15th International Congress of Actuaries, vol. 2, pp. 433-443, 1957.

[14] X. S. Lin, G. E. Willmot, and S. Drekic, "The classical risk model with a constant dividend barrier: analysis of the GerberShiu discounted penalty function," Insurance: Mathematics \& Economics, vol. 33, no. 3, pp. 551-566, 2003.

[15] D. C. M. Dickson and H. R. Waters, "Some optimal dividends problems," Astin Bulletin, vol. 34, no. 1, pp. 49-74, 2004.

[16] S. Li and Y. Lu, "Moments of the dividend payments and related problems in a Markov-modulated risk model," North American Actuarial Journal, vol. 11, no. 2, pp. 65-76, 2007.

[17] W. G. Yu, "Some results on absolute ruin in the perturbed insurance risk model with investment and debit interests," Economic Modelling, vol. 31, no. 1, pp. 625-634, 2013.

[18] W. G. Yu, "Randomized dividends in a discrete insurance risk model with stochastic premium income," Mathematical Problems in Engineering, vol. 2013, Article ID 579534, 9 pages, 2013.

[19] W. G. Yu, "On the expected discounted penalty function for a Markov regime-switching insurance risk model with stochastic premium income," Discrete Dynamics in Nature and Society, vol. 2013, Article ID 320146, 9 pages, 2013.

[20] D. J. Yao, H. L. Yang, and R. M. Wang, "Optimal risk and dividend control problem with fixed costs and salvage value: variance premium principle," Economic Modelling, vol. 37, pp. 53-64, 2014.

[21] J. Zhu, "Optimal dividend control for a generalized risk model with investment incomes and debit interest," Scandinavian Actuarial Journal, vol. 2013, no. 2, pp. 140-162, 2013.

[22] J. Y. Tan, P. T. Yuan, Y. J. Cheng, and Z. Q. Li, “An optimal dividend strategy in the discrete Sparre Andersen model with bounded dividend rates," Journal of Computational and Applied Mathematics, vol. 258, pp. 1-16, 2014.

[23] H. U. Gerber and E. S. W. Shiu, "On the time value of ruin," North American Actuarial Journal, vol. 2, no. 1, pp. 48-78, 1998.

[24] X. S. Lin and G. E. Willmot, "The moments of the time of ruin, the surplus before ruin, and the deficit at ruin," Insurance: Mathematics \& Economics, vol. 27, no. 1, pp. 19-44, 2000.

[25] D. C. M. Dickson and C. Hipp, "On the time to ruin for Erlang(2) risk processes," Insurance: Mathematics \& Economics, vol. 29, no. 3, pp. 333-344, 2001.

[26] X. H. Zhao and C. C. Yin, "Ruin problems for a Sparre Andersen risk model," Journal of Qufu Normal University, vol. 31, no. 2, pp. 9-12, 2005. 


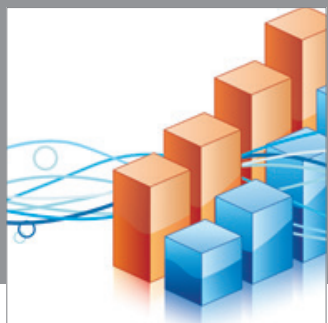

Advances in

Operations Research

mansans

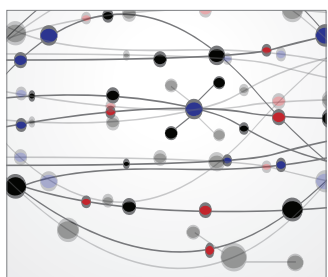

The Scientific World Journal
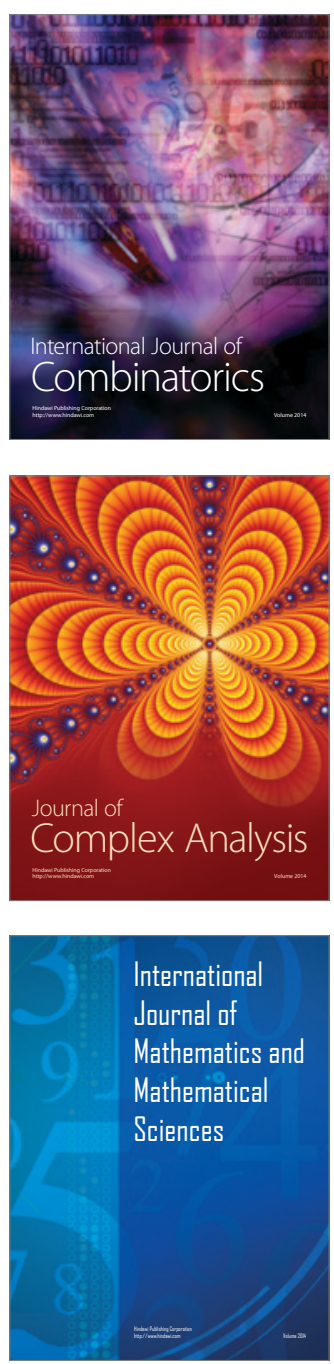
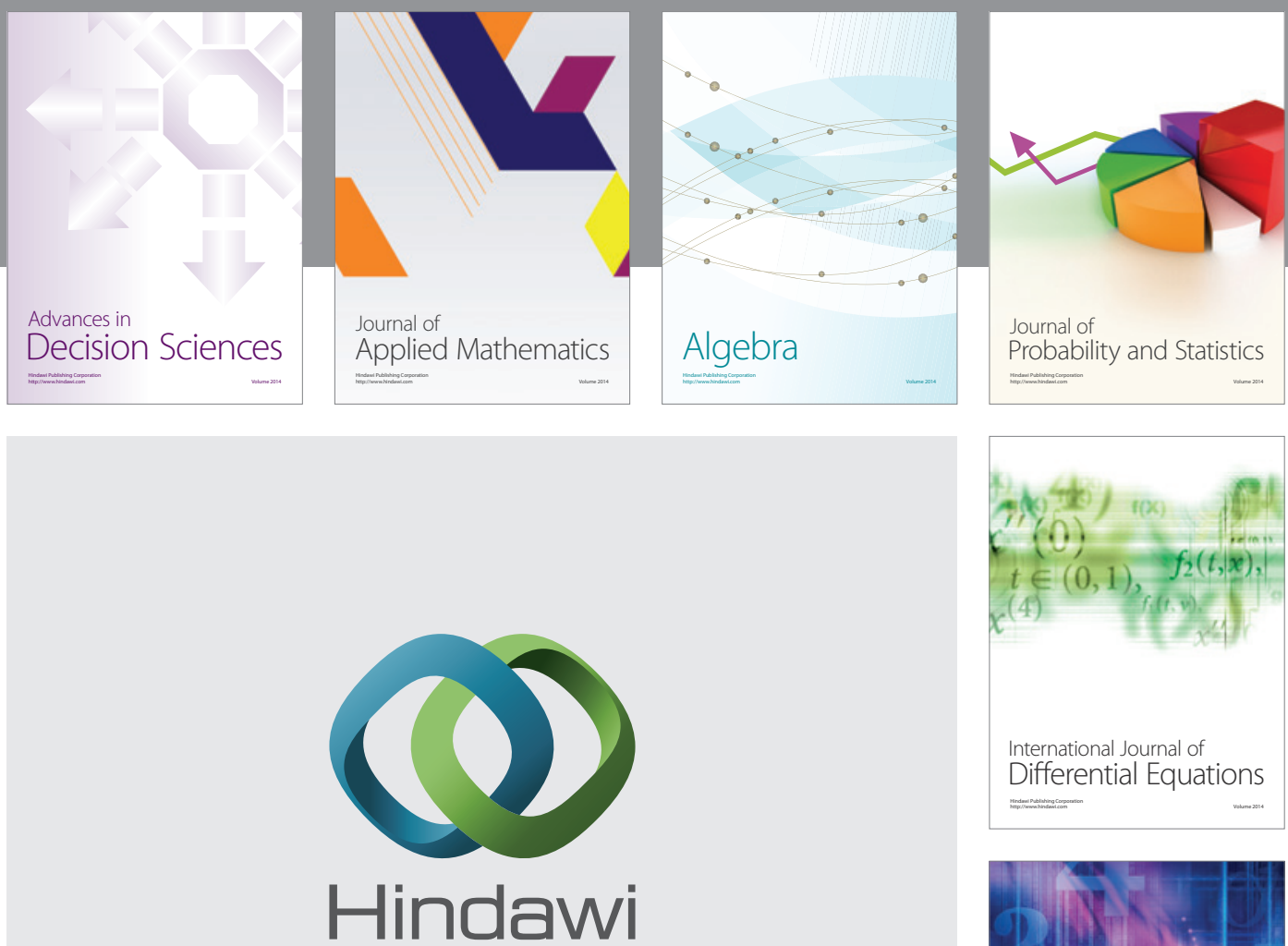

Submit your manuscripts at http://www.hindawi.com
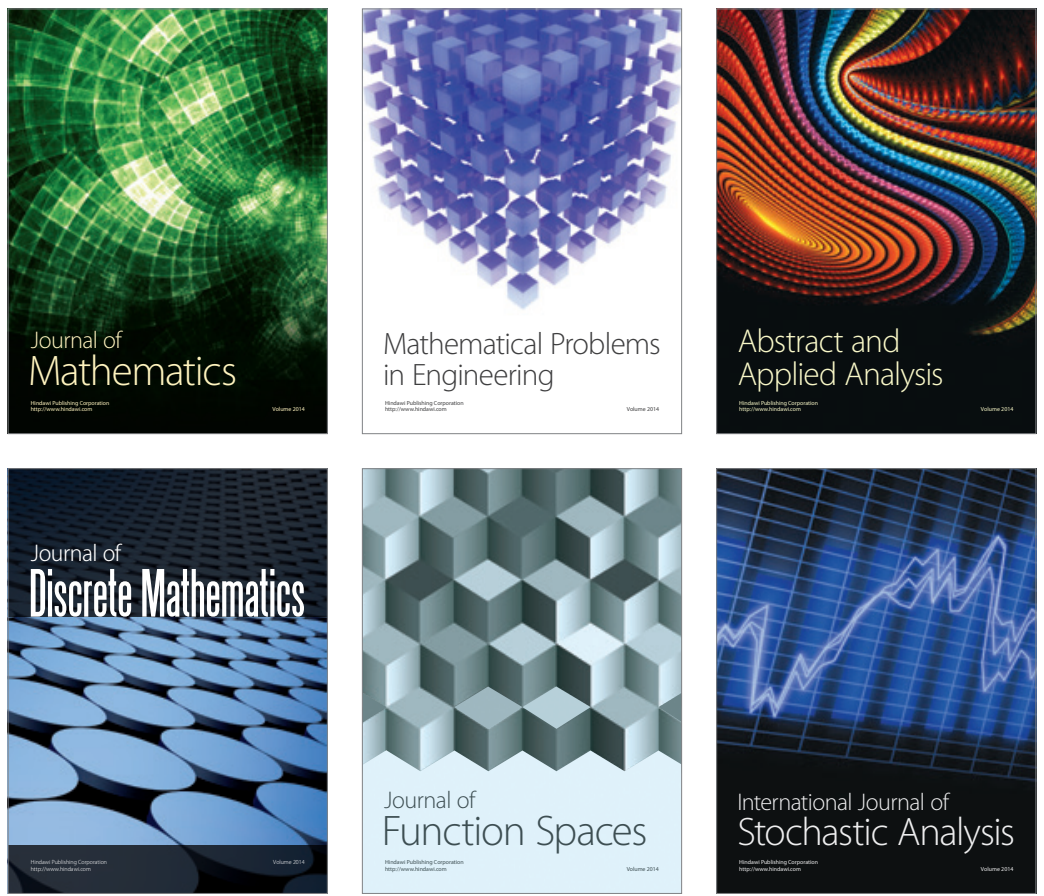

Journal of

Function Spaces

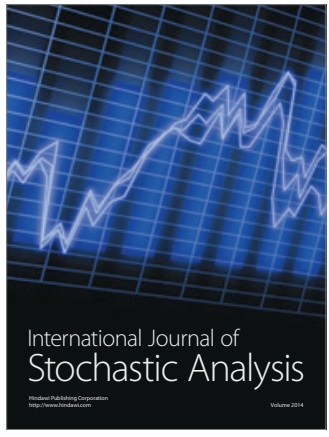

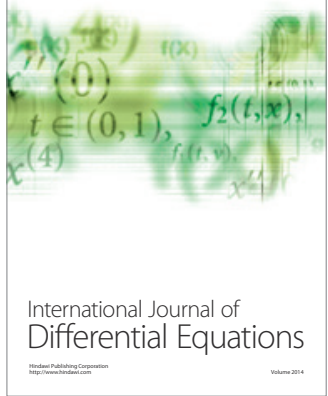
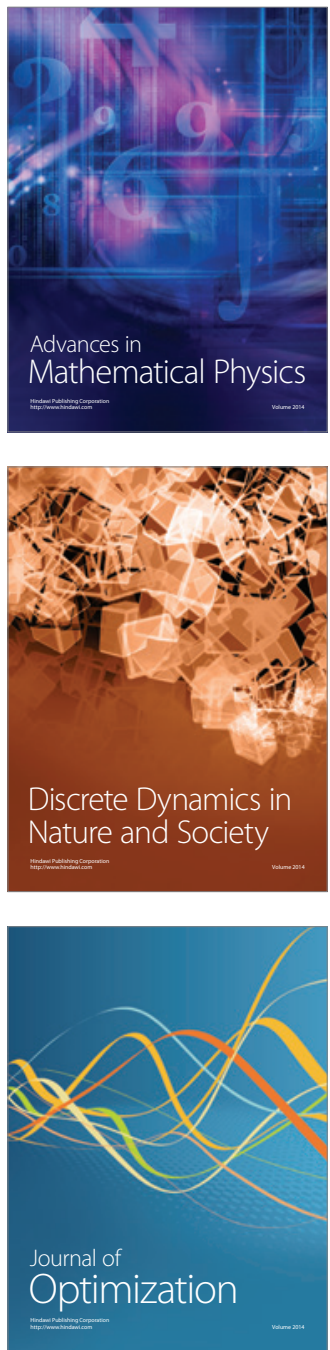